\title{
Fish Oil/Glycerol/Egg Lecithin-based Emulsion
}

National Cancer Institute

\section{Source}

National Cancer Institute. Fish Oil/Glycerol/Egg Lecithin-based Emulsion. NCI Thesaurus.

Code C104273.

An injectable, nutritional lipid emulsion composed of $10 \%$ fish oil and high amounts of the fish oil-derived polyunsaturated omega-3 fatty acids eicosapentaenoic acid (EPA) and docosahexaenoic acid (DHA). Additionally, the fish oil/glycerol/egg lecithin-based emulsion contains, myristic acid, palmitic acid, palmitoleic acid, stearic acid, oleic acid, linoleic acid, linolenic acid, octadecatetraenoic acid, eicosaenoic acid, arachidonic acid, docosaenoic acid, and docosapentaenoic acid. This agent supplies essential fatty acids that can be incorporated into cell membranes. The fatty acids may decrease the production of certain pro-inflammatory cytokines, including interleukin 1 (IL-1), IL-6 and tumor necrosis factor (TNF). In addition to fish oil, this lipid emulsion contains egg phospholipids to maintain membrane integ rity; glycerol to provide energy through glycolysis; and the antioxidant alpha-tocopherol (vitamin E). 